\title{
Antimicrobial Activities Of Polyphenol Extract Of Peanut Skins
}

\author{
Wen-xu XIA ${ }^{1}$, Ping ZHAO ${ }^{2, *}$, Jia-bin $\mathrm{WANG}^{3}$, Zhan-juan $\mathrm{LI}^{2}$ and \\ Nanju Alice LEE ${ }^{4}$ \\ ${ }^{1}$ College of Food Science and Technology, Shanghai Ocean University, Shanghai \\ 201306, China \\ ${ }^{2}$ School of Life Science \& Engineering, Lanzhou University of Technology, Lanzhou \\ 730050, China \\ ${ }^{3}$ College Of Information Engineering, Shanghai Maritime University, Shanghai 201306, \\ China \\ ${ }^{4}$ School of Chemical Sciences and Engineering, Faculty of Engineering, UNSW \\ SYDNEY NSW 2052, Australia \\ ${ }^{*}$ Corresponding author
}

Keywords: Peanut Testa, Polyphenol Extracts, Antimicrobial Activity, Minimum Inhibitory Concentration, Equivalent Against Benzylpenicillin Sodium.

\begin{abstract}
In this study, the cardinal and purple peanut testa were used as the raw materials for extracting polyphenol substances with ultrasonic and freeze-drying. Then the bacteriostasis and antifungal activities of the extracts were investigated by the methods of disc diffussion and equivalent against the Benzylpenicillin Sodium to certain bacteria. The results showed that, at the same concentration, bacteriostatic activity of the crude extract of cardinal peanut testa was stronger than that of purple peanuts. The inhibitory concentrations of crude extracts were up to $0.5 \mathrm{~g} / \mathrm{L}$ for cardinal peanut testa, $1 \mathrm{~g} / \mathrm{L}$ for purple peanut testa and equivalents against the Benzylpenicillin Sodium were $6000 \mathrm{U}$ and $6400 \mathrm{U}$, respectively.
\end{abstract}

\section{Introduction}

Polyphenol, which could be divided into simple phenols, phenolic acids, hydroxycinnamic acids and flavonoids, was a general name of a class of substances possessing an aromatic ring, combinated by multiple hydroxyl and existed widely in fruits and vegetables[1]. Polyphenols could enhance the biological activity of insulin, hypoglycemic, and many other bioactivity, such as antioxidant activity[2-4], antitumor, antimicrobial, and antiviral activity[5].

Peanuts are abundant in China. China not only made up to $1 / 3$ peanuts production of the world, but also exported the most. As a by-product of peanut products, many polyphenols compounds materials with very important physiology function, however, cardinal and purple peanut testas have not been used effectively and sufficiently, so it is highly profitable and profoundly remarkable to extract and refine the natural active components from peanut testa. And the extraction of natural active ingredient in them had good economic benefit and profound significance. In this study, the cardinal and purple peanut testa was used as the raw materials for extracting polyphenol substances with ultrasonic and freeze-drying. And the bacteriostasis and antifungal activities of the extracts were investigated by the methods of disc diffussion and equivalent against the Benzylpenicillin Sodium to certain bacteria. 


\section{Materials and Methods}

\section{Materials and Reagents}

Cardinal peanut skins were provided by Shandong Luhua Co.; Enriched-Se peanuts were from Sichuan Nanmu grass industry Co., Ltd..

Anhydrous ethanol was from Laiyang Shuangshuang Chemical Co., Ltd. (Shandong, China). Benzylpenicillin Sodium for injection was from Harbin Pharmaceutical Group Co., Ltd. (China). Petroleum ether, sodium chloride, and the other chemicals used were of analytically grade.

\section{Strains}

Six different strains were used: Escherichia coli (Migula) Castellani et Chalmers (GSICC 30507), Staphylococcus aureus subsp. Aureeus Rosenbach (GSICC 31902), Bacillus subtilis (GSICC 30212), Penicillium sp. (CICC40361), Aspegillus niger (CICC 2039), and Actinomucor sp. (CICC 40252). The first two were obtained from Gansu Microbiology Save Center, China, while the others were provided by School of Life Science and Engineering of Lanzhou University of Technology.

\section{Preparation of Polyphenol Extract Of Peanut Testa}

Enriched-Se peanuts were hand-shelled, adjusted to $80^{\circ} \mathrm{C}$ in a convection oven for $1 \mathrm{~h}$ before removing the purple testa. The peanut testas of two types were pulverized using an electric grinder, sifted with 40 mesh sieve, degreased by petroleum ether and stored in refrigerated at $4^{\circ} \mathrm{C}$.

The polyphenol extracts of the two types of peanut testas were extracted used a Ultrasonic Cell Crusher (JY92- II, Ningbo Scientz Biotechnology Co., Ltd., China) as the following conditions: extraction power, $320 \mathrm{~W}$; extraction time, $10 \mathrm{~min}$; liquid to solid ratio, 16:1; ethanol concentration, $75 \%$. The extraction solution was centrifuged using a flying Pigeon desktop centrifuge (TDL-5-A, ShangHai Anting Scientific Instrument Factory, China) at $3500 \times g$ for $10 \mathrm{~min}$. After centrifugation, the residue were extracted for once more adjusted to liquid to solid ratio of $9: 1$, centrifuged under the same condition, and then combined the extraction solution. The extraction solution was evaporated under vacuum in a rotary evaporation (RE52-86A, ShangHai Yarong Biochemical Instrument Factory, China). The extracts were freeze-dried using a freeze dryer (FD-1-55, Beijing Boyikang experimental apparatus Co., Ltd., China), the freeze temperature is $-40^{\circ} \mathrm{C}$ and the time of lyophilization is $20 \sim 24 \mathrm{~h}$, and the cardinal peanut testa and purple peanut testa extracts were obtained.

\section{Antimicrobial Activity}

\section{Activation of Bacteria Strains}

E. coli, S. aureus were activated with MRS agar media (g/L: beef extract 3, peptone 10, agar 20, $\mathrm{NaCl}$ 5, water 1000; pH 7.0 7.5), while Bacillus subtilis, Penicillium sp., Aspegillus niger, and Actinomucor sp. with PDA medium slants (g/L: pototal 200, glucose 20, agar 20, water 1000). All bacterial cultures were incubated in aerobic conditions and all of the measurements were carried out under aseptic conditions ${ }^{[6]}$. 


\section{Preparation of Suspension of Strains}

$200 \mathrm{~mL}$ of physiological saline were inoculated into the six slants tubes, and then removed the suspension of strains into six flasks sterilized after stiring thoroughly. A physiological saline was used to dilute the suspension of strains, until the final inoculum obtained was $10^{6}-10^{8} \mathrm{CFU} / \mathrm{mL}$.

\section{Determination of Antimicrobial Activity}

$0.3 \mathrm{~g}$ of cardinal and purple peanut testas freeze-dried were dissolved in $15 \mathrm{~mL}$ sterile water and diluted, respectively. 20, 4, 0.8, 0.16 and $0.032 \mathrm{~g} / \mathrm{L}$ of solution were obtained. $0.75 \mathrm{~g}$ cardinal and purple peanut testas freeze-dried were dissolved in $15 \mathrm{~mL}$ sterile water and diluted, respectively. 50, 10, 2, 0.4, 0.08, 0.016 and $0.0032 \mathrm{~g} / \mathrm{L}$ of solution were obtained. With $0.85 \%$ sterile saline as control, antimicrobial activities of each solution were measured according to the disc diffusion method described at literature ${ }^{[7]}$. Sterile paper discs of $6 \mathrm{~mm}$ diameter were used throughout this experiment. All measurements were done in triplicate, and the bacteriostatic values were expressed as mean.

\section{Determination of Minimum Inhibitory Concentration (MIC)}

A concentration series solution $(0.25,0.5,1,2$, and $3 \mathrm{~g} / \mathrm{L}$, respectively) in sterile water of cardinal and purple peanut testas and $0.85 \%$ sterile saline were used to determinate the MIC with the disc diffusion method. The MIC was considered the lowest concentration of the extracts that inhibited the bacterial or the fungic growth, after incubation. All measurements were done in triplicate, and the bacteriostatic values were expressed as mean.

\section{Antimicrobial Activity of Antibiotics and Determination of Equivalent}

A bottle of Benzylpenicillin Sodium (800 000 U, $0.48 \mathrm{~g}$ ) was dissolved in sterile water and diluted, a concentration series solution was obainted, respectively, 80×104, 16×104, $3.2 \times 104,0.64 \times 104,0.128 \times 104$, and $0.0256 \times 104 \mathrm{U}$ of Benzylpenicillin Sodium. With $0.85 \%$ sterile saline as control, antimicrobial activity of Benzylpenicillin Sodium and antimicrobial equivalent was determinated. All measurements were done in triplicate, and the bacteriostatic values were expressed as mean.

\section{Results and Discussion}

\section{Antimicrobial Activity}

Results show that polyphenol extracts of cardinal peanut testas have effective antimicrobial activity against all studied strains. The antimicrobial activity is more significant aginst S. aureus, Bacillus subtilis, Aspegillus niger, and Actinomucor sp. than against E. coli, and Penicillium sp., which produces significant zone of inhibition at the concentration of $0.8 \mathrm{~g} / \mathrm{L}$ or more (Table 1 ).

Table 1. Antimicrobial activity of polyphenol extracts of cardinal peanut testa

\begin{tabular}{|c|c|c|c|c|c|c|c|c|c|c|}
\hline \multirow[b]{2}{*}{ strains } & \multicolumn{10}{|c|}{ Zone of inhibition (mm) } \\
\hline & $\mathrm{A}^{1}$ & $\mathrm{~B}^{1}$ & $\mathrm{C}^{1}$ & $\mathrm{D}^{1}$ & $E^{1}$ & $\mathrm{~F}^{1}$ & $\mathrm{G}^{1}$ & $\mathrm{H}^{1}$ & $I^{1}$ & $\mathrm{~J}^{1}$ \\
\hline E.coli & $17.5 \pm 0.15$ & $16.5 \pm 0.1$ & $14.4 \pm 0.25$ & $13.4 \pm 0.2$ & $12.1 \pm 0.1$ & $11.0 \pm 0.1$ & - & - & - & - \\
\hline S. aureus & $19.0 \pm 0.4$ & $17.0 \pm 0.1$ & $14.6 \pm 0.2$ & $13.6 \pm 0.1$ & $13.0 \pm 0.4$ & $11.4 \pm 0.1$ & - & - & - & - \\
\hline Bacillus subtilis & $18.1 \pm 0.2$ & $16.2 \pm 0.1$ & $14.3 \pm 0.1$ & $13.2 \pm 0$ & $12.9 \pm 0.2$ & $11.1 \pm 0.2$ & - & - & - & - \\
\hline Penicillium sp. & $17.4 \pm 0.1$ & $16.7 \pm 0.3$ & $14.3 \pm 0.1$ & $13.4 \pm 0.3$ & $12.9 \pm 0.2$ & $11.3 \pm 0.3$ & - & - & - & - \\
\hline Actinomucor sp. & $19.5 \pm 0.06$ & $17.5 \pm 0.06$ & $14.7 \pm 0.15$ & $13.7 \pm 0.15$ & $13.1 \pm 0.15$ & $11.4 \pm 0.1$ & - & - & - & - \\
\hline Aspegillus niger & $18.6 \pm 0.06$ & $17.2 \pm 0.2$ & $14.5 \pm 0.15$ & $13.3 \pm 0.1$ & $12.8 \pm 0.06$ & $11.2 \pm 0.15$ & - & - & - & - \\
\hline
\end{tabular}


Zone of inhibition is directly proportional to the concentration of polyphenol extracts, in other words, antimicrobial activity of polyphenol extracts of cardinal peanut testa is positively correlated to the concentration of extracts.

Results show that polyphenol extracts of cardinal peanut testas have effective antimicrobial activity against all studied strains. The antimicrobial activity is more significant aginst $S$. aureus, E. coli, Penicillium sp. and Aspegillus niger, than aginst Bacillus subtilis, and Actinomucor sp., which produces significant zone of inhibition at the concentration of $2.0 \mathrm{~g} / \mathrm{L}$ or more (Table 2).

Table 2. Antimicrobial activity of polyphenol extracts of purple peanut testa

\begin{tabular}{|c|c|c|c|c|c|c|c|c|c|}
\hline \multirow{2}{*}{ strains } & \multicolumn{9}{|c|}{ Zone of inhibition (mm) } \\
\hline & $\mathrm{A}^{2}$ & $\mathrm{~B}^{2}$ & $\mathrm{C}^{2}$ & $\mathrm{D}^{2}$ & $E^{2}$ & $\mathrm{~F}^{2}$ & $\mathrm{G}^{2}$ & $\mathrm{H}^{2}$ & $\mathrm{I}^{2}$ \\
\hline E.coli & $15.3 \pm 0.1$ & $14.2 \pm 0.12$ & $13.2 \pm 0.2$ & $12.4 \pm 0.06$ & $11.2 \pm 0.1$ & - & - & - & - \\
\hline S. aureus & $15.8 \pm 0.15$ & $14.9 \pm 0.15$ & $13.5 \pm 0.25$ & $12.8 \pm 0.1$ & $11.5 \pm 0.06$ & - & - & - & - \\
\hline Bacillus subtilis & $15.2 \pm 0.06$ & $14.3 \pm 0.15$ & $13.2 \pm 0$ & $12.2 \pm 0.1$ & $11.2 \pm 0.1$ & - & - & - & - \\
\hline Penicillium sp. & $15.3 \pm 0.06$ & $14.2 \pm 0.12$ & $13.3 \pm 0.1$ & $12.2 \pm 0.15$ & $11.3 \pm 0.15$ & - & - & - & - \\
\hline Actinomucor sp. & $15.2 \pm 0.12$ & $14.1 \pm 0.06$ & $13.3 \pm 0.15$ & $12.3 \pm 0.2$ & $11.2 \pm 0.12$ & - & - & - & - \\
\hline Aspegillus niger & $15.3 \pm 0.1$ & $14.2 \pm 0.06$ & $13.1 \pm 0.06$ & $12.5 \pm 0.06$ & $11.3 \pm 0.12$ & - & - & - & - \\
\hline
\end{tabular}

Zone of inhibition varies directly as the concentration of polyphenol extracts, in other words, antimicrobial activity of polyphenol extracts of purple peanut testa is positively correlated to the concentration of extracts.

Benzylpenicillin Sodium has effective antimicrobial activity aginst E. coli, $S$. aureus, and Bacillus subtilis, but Penicillium sp., Aspegillus niger, and Actinomucor sp., and it most significant aginst Bacillus subtilis, which produces significant zone of inhibition at tl Table 3. Antimicrobial activity of Benzylpenicillin Sodium

\begin{tabular}{cccccccc}
\hline \multirow{2}{*}{ Strains } & \multicolumn{7}{c}{ Zone of inhibition $(\mathrm{mm})$} \\
\cline { 2 - 7 } & $\mathrm{A}^{3}$ & $\mathrm{~B}^{3}$ & $\mathrm{C}^{3}$ & $\mathrm{D}^{3}$ & $\mathrm{E}^{3}$ & $\mathrm{~F}^{3}$ & $\mathrm{G}^{3}$ \\
\hline E.coli & $20.4 \pm 0.1$ & $15.7 \pm 0.06$ & $12.5 \pm 0.15$ & $11.3 \pm 0.15$ & - & - & - \\
S. aureus & $20.3 \pm 0.25$ & $16.6 \pm 0.15$ & $13.7 \pm 0.06$ & $11.9 \pm 0.2$ & - & - & - \\
Bacillus subtilis & $21.5 \pm 0.35$ & $17.5 \pm 0.06$ & $14.2 \pm 0.06$ & $12.3 \pm 0.15$ & - & - & - \\
Penicillium sp. & - & - & - & - & - & - & - \\
Actinomucor sp. & - & - & - & - & - & - & - \\
Aspegillus niger & - & - & - & - & - & - & - \\
\hline $\mathrm{A}^{3}-\mathrm{G}^{3}$ are the concentration of polyphenol extracts of cardinal peanut testa, $80 \times 10^{4}, 16 \times 10^{4}, 3.2 \times 10^{4}$, \\
$0.64 \times 10^{4}, 0.128 \times 10^{4}, 0.0256 \times 10^{4}$, and 0 U, respectively. \\
- No zone of inhibition
\end{tabular}

Zone of inhibition is in proportion with the concentration of Benzylpenicillin Sodium, in other words, antimicrobial activity of polyphenol extracts of Benzylpenicillin Sodium is positively correlated to the concentration of extracts.

\section{Minimum Inhibitory Concentration}

As shown in Table 4 and 5, MIC values are $0.5 \mathrm{~g} / \mathrm{L}$ and $1.0 \mathrm{~g} / \mathrm{L}$ for the extracts of Cardinal peanut testas and purple peanut testas against E. coli, S. aureus, Bacillus subtilis, Penicillium sp., Aspegillus niger, and Actinomucor sp., respectively.

Table 4. MIC of polyphenol extracts of cardinal peanut testa

\begin{tabular}{ccccccc}
\hline \multirow{2}{*}{ strains } & \multicolumn{5}{c}{ Zone of inhibition (mm) } \\
\cline { 2 - 6 } & $\mathrm{A}^{4}$ & $\mathrm{~B}^{4}$ & $\mathrm{C}^{4}$ & $\mathrm{D}^{4}$ & $\mathrm{E}^{4}$ & $\mathrm{~F}^{4}$ \\
\hline E.coli & $12.7 \pm 0.12$ & $12.1 \pm 0.06$ & $11.5 \pm 0.06$ & - & - & - \\
S. aureus & $13.5 \pm 0.1$ & $13.0 \pm 0.15$ & $12.2 \pm 0.06$ & - & - & - \\
Bacillus subtilis & $13.1 \pm 0.06$ & $12.9 \pm 0.25$ & $12.0 \pm 0.25$ & - & - & - \\
Penicillium sp. & $13.2 \pm 0.06$ & $12.9 \pm 0.15$ & $11.9 \pm 0.2$ & - & - & - \\
Actinomucor sp. & $13.4 \pm 0.15$ & $13.1 \pm 0$ & $12.4 \pm 0.15$ & - & - & - \\
Aspegillus niger & $13.0 \pm 0.06$ & $12.8 \pm 0.25$ & $11.7 \pm 0.1$ & - & - & - \\
\hline A $-F^{4}$ are the concentration of polyphenol extracts of cardinal peanut testa, 3, 2, $1,0.5,0.25$, and 0 \\
g/L, respectively. \\
- No zone of inhibition
\end{tabular}


Table 5. MIC of polyphenol extracts of purple peanut testa

\begin{tabular}{ccccccc}
\hline \multirow{2}{*}{ strains } & \multicolumn{7}{c}{ Zone of inhibition $(\mathrm{mm})$} \\
\cline { 2 - 6 } & $\mathrm{A}^{5}$ & $\mathrm{~B}^{5}$ & $\mathrm{C}^{5}$ & $\mathrm{D}^{5}$ & $\mathrm{E}^{5}$ & $\mathrm{~F}^{5}$ \\
\hline E.coli & $11.8 \pm 0.15$ & $11.2 \pm 0.06$ & - & - & - & - \\
S. aureus & $12.3 \pm 0.06$ & $11.5 \pm 0.12$ & - & - & - & - \\
Bacillus subtilis & $11.9 \pm 0.06$ & $11.2 \pm 0.1$ & - & - & - & - \\
Penicillium $\mathrm{sp}$. & $11.7 \pm 0.12$ & $11.3 \pm 0.2$ & - & - & - & - \\
Actinomucor $\mathrm{sp}$. & $11.8 \pm 0.2$ & $11.2 \pm 0.15$ & - & - & - & - \\
Aspegillus niger & $12.0 \pm 0.1$ & $11.3 \pm 0.06$ & - & - & - & - \\
\hline
\end{tabular}

\section{EAPS}

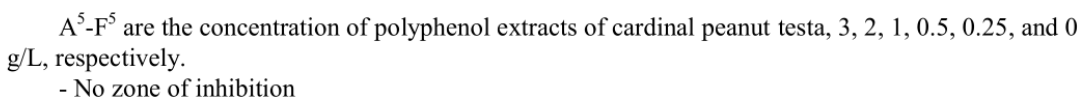

As can be noted in Table 3, 4, and 5, it can be brought to the upshort that the minimum equivalent values against Benzylpenicillin Sodium of cardinal and purple peanut testas are $6000 \mathrm{U}$ and $6400 \mathrm{U}$, respectively.

\section{Conclusions}

Cardinal and purple peanut testas polyphenol produced significant zone of inhibition at the concentration of 0.8 and $2.0 \mathrm{~g} / \mathrm{L}$ or more, while the concentration of Benzylpenicillin Sodium was more than $0.64 \times 104$ U. The bacteriostatic activity varied of the two types peanut testas/Benzylpenicillin Sodium directly with the concentration of the extract.

MIC values are $0.5 \mathrm{~g} / \mathrm{L}$ and $1.0 \mathrm{~g} / \mathrm{L}$ for the extracts of cardinal peanut testas and purple peanut testas against E. coli, S. aureus, Bacillus subtilis, Penicillium sp., Aspegillus niger, and Actinomucor sp., respectively. The minimum equivalent values against Benzylpenicillin Sodium of cardinal and purple peanut testas are $6000 \mathrm{U}$ and $6400 \mathrm{U}$, respectively. In a word, polyphenol extract of peanut testas exhibits broad-spectrum antimicrobial activity.

\section{Acknowledgement}

This work was partially supported by the STATE CSHOLARSHIP FUND AWARD of CSC (No.200421G074) and the Science Foundation of the Gansu Province, China (Grant No.0803RJZA044).

\section{References}

[1] Kevin Robards, Paul D. Prenzler, Greg Tucker, Prasan Swatsitang, and William Glover, Phenolic compounds and their role in oxidative processes in fruits, Food Chem. 66 (1999) 401-436.

[2] Vinson, J., Jang, J., Dabbagh, Y. A., Serry, M. M., and Cai, S., Plant polyphenols exhibit lipoprotein-bound antioxidant activity using an in vitro model for heart disease, Journal of Agricultural and Food Chemistry. 43 (1995) 2798-2799.

[3] Baldioli, M., Servili, M., Perretti, G., and Montedoro, G. F., Antioxidant activity of tocopherols and phenolic compounds of virgin olive oil, Journal of the American Oil Chemists' Society. 73 (1996) 1589-1593.

[4] Visioli, F., Bellomo, G., and Galli, C., Free radical-scavenging properties of olive oil polyphenols, Biochemical and Biophysical Research Communications. 247 (1998) 60-64. 
[5] Norberta W. Schoene, Meghan A. Kelly, Marilyn M. Polansky, and Richard A. Anderson, Water-soluble polymeric polyphenols from cinnamon inhibit proliferation and alter cell cycle distribution patterns of hematologic tumor cell lines, Cancer Letters. 230 (2005) 134-140.

[6] Smânia, A., Jr., Monache, F. D., Smânia, E. F., Gil, M. L., Benchetrit, L. C., and Cruz, F. S., Antibacterialactivityofasubstanceproduced by the fungus Pycnoporus sanguineus (Fr.) Murr, Journal of Ethnopharmacology, Inglaterra. 45 (1995) 177-181.

[7] Bauer, A. W., Kirby, M. D. K., Sherries, J. C., and Truck, M., Antibiotic susceptibilities testing by standard single disc diffusion method, American Journal of Clinical Pathology. 45 (1966) 493-496. 